\title{
Characteristics of Blood Oxygen Level-Dependent and Diffusion- Weighted Magnetic Resonance Imaging in Tubulointerstitial Nephritis: an initial experience
}

Tao Su ( $\square$ tao.su@bjmu.edu.cn )

Peking University First hospital https://orcid.org/0000-0002-6857-8146

Xuedong Yang

China Academy of Chinese Medical Sciences Guanganmen Hospital

Rui Wang

Peking University First Hospital

Li Yang

Peking University first hospital

Xiaoying Wang

Peking University first hospital

\section{Research article}

Keywords: Magnetic resonance imaging, Blood oxygenation level-dependent, Diffusion-weighted, tubulointerstitial nephritis

Posted Date: August 25th, 2020

DOI: https://doi.org/10.21203/rs.3.rs-50263/v1

License: (1) This work is licensed under a Creative Commons Attribution 4.0 International License. Read Full License 


\section{Abstract}

Diffusion weighted (DW) and blood oxygen level-dependent (BOLD) magnetic resonance (MR)imaging are basic classical sequences of functional MR (fMR) in clinical application, but the exploration in the field of non-transplanted kidney disease is limited.

Objects

To analyze the characteristics of global apparent diffusion coefficient (ADC) values and renal oxygenation status by $R_{2}$ * values using DW and BOLD imaging in patients with acute, chronic tubulointerstitial nephritis (ATIN, CTIN) and healthy control.

Methods

Four biopsy-proven ATIN, thirteen clinical CTIN patients in stage 2-5 of chronic kidney disease and four controls were enrolled. They underwent fMR imaging with a 3.0-T MR scanner. A multiple gradient-echo sequence was used to acquire 12 T2*-weighted images for calculation of $R_{2}{ }^{*}$ map. DW imaging was acquired by combining a single-shot spin-echo echo planar imaging pulse sequence and the additional motion probing gradient pulses along the $\mathrm{x}, \mathrm{y}$, z-axes. We used two different $b$ value groups: 0 and $200 \mathrm{~s} / \mathrm{mm}^{2}$ as well as 0 and $800 \mathrm{~s} / \mathrm{mm}^{2}$. For ATIN patients, DW and BOLD MR were performed at the time of renal biopsy (T0) and the third month (T3). Serum creatinine levels at the T3 and sixth month (T6) were regarded as indicators of long-term renal prognosis. Pathological changes such as tubular injury, tissue edema, severity of interstitial inflammation or fibrosis were assessed semi-quantitatively. Activity index ( $\mathrm{Al})$ and chronic index (Cl) were calculated. Correlation analysis were conducted within MR parameters, pathological and clinical indexes.

Results

In ATIN kidneys, ADCs were significantly lower than control, and showed an obvious remission through three months (both $b$ values, $\mathrm{p}<0.05)$. Both cortical $R_{2}$ * values $\left(\mathrm{CR}_{2}{ }^{*}\right)$ and medullary $\mathrm{R}_{2}$ * values $\left(\mathrm{MR}_{2}{ }^{*}\right)$ were decreased, the difference was significant in the change of $M R_{2}{ }^{*}$. $A$ rapid recovery of $\mathrm{MR}_{2}{ }^{*}$ was also observed at T3. There was no relationship between $\mathrm{fMR}$ parameters and histopathological indexes (whether compared separately or as $\mathrm{Al}$ and $\mathrm{Cl})$. $\mathrm{MR}_{2}{ }^{*}$ had a close relationship with eGFR ( $\left.\mathrm{R}=0.682, \mathrm{P}=0.001\right)$. The change of $\mathrm{ADCs}(\triangle \mathrm{ADC})$ when $b$ value was $0,200 \mathrm{~s} / \mathrm{mm}^{2}(R=-0.956, P=0.044)$ and $0,800 \mathrm{sec} / \mathrm{mm}^{2}(R=-0.968, P=0.032)$ were inversely correlated to $A D C s, \Delta M R 2(R=0.979, P=0.021)$ and pathological $\mathrm{Cl}(\mathrm{R}=-0.977, \mathrm{P}=0.023)$. Renal long-term prognosis analysis among candidate predictive markers showed no relationship with timepoint $A D C$ or $R_{2}$ * values, but $\triangle M R_{2}$ * had a significant correlation to $S c r$ levels at $T 3(R=-0.959, P=0.041)$ and $T 6(R=0.98, P=0.02)$. That was, the lower the $A D C$ value ( $b$ was $0,200 \mathrm{sec} / \mathrm{mm}^{2}$ ), the greater the increase of $A D C$ and $M_{2}{ }^{*}$ in the next three months, then the subsequent Scr level would be lower. In CTIN group, a low level of $\mathrm{MR}_{2}$ * was observed while $\mathrm{CR}_{2}$ * remained unchanged.

Conclusions

Direct evidence of global ADCs and renal oxygenation were got in TIN patients for the first time. $\mathrm{MR}_{2}$ * served as a promising marker reflecting eGFR. A lower ADC value when $b$ was $0,200 \mathrm{sec} / \mathrm{mm}^{2}$ was a predictive marker to reversible acute injury. The "pseudo normalization" of $\mathrm{CR}_{2}$ * in CTIN might be the result of the aggravation of renal ischemic changes, contributing to the progression of CKD.

\section{Background}

Functional magnetic resonance (fMR) imaging has recently grown to be a useful tool to evaluate real-time renal function[1]. The functional MR sequences mainly include blood oxygen level-dependent (BOLD), diffusion-weighted (DW) imaging, arterial labeling perfusion (ASL) and dynamic contrast-enhanced imaging (DCl). They provide information about diffusion, perfusion, and oxygenation of kidneys besides morphological parameters. These novel techniques serve as promising markers helping to further understand pathophysiology of acute kidney injury (AKI) and chronic kidney disease (CKD)[2-6]. Functional MR is also recommended for early differentiation diagnosis of renal dysfunction after kidney transplantation[7-8]. Researches with fMR were still exploratory in animal models of AKI and some human observational studies[9-11], in human studies of CKD trying to compare MR parameters with renal pathological index[3,9]. It can be seen that the characteristics of $\mathrm{fMR}$ in nontransplantation human kidney diseases by contrast, were poorly understood yet.

DW and BOLD from magnetic resonance imaging are early used techniques. The apparent diffusion coefficient (ADC) calculated from DW images was influenced by both pure diffusion and perfusion-dependent diffusion at a low $b$ value. The value was observed to decrease associated with severity of renal dysfunction and degree of renal fibrosis after AKI[12]. But in another CKD rat model, ADC values could not reflect degree of kidney fibrosis[10]. BOLD MR imaging was demonstrated to effectively detect changes in intra-renal oxygenation by measuring the $\mathrm{R}_{2}$ * levels of the renal cortex and medulla[9]. Li et al. observed the immediate increase in $\mathrm{R}_{2}$ * in the renal inner stripe of the outer medulla after the injection of contrast agent, which suggesting existence of tissue hypoxia probably induced by hypoperfusion[13], that BOLD served as an earliest biomarker of contrast induced AKI. In diabetes patients, it was found that $R_{2}$ * value was decreased after single dose of furosemide 
injection as a result of increased oxygen consumption of tubular Na-K transporter working. While in another report, Renal BOLD-MRI was found not reflecting renal function CKD [14]. Furthermore, intra-renal change of oxygenation contributes to progression of chronic kidney disease[15]. This shows that both $A D C$ and $\mathrm{R}_{2}$ * value might be influenced by intra-renal perfusion, tubular injury, interstitial inflammation or fibrosis, all these pathological changes that resulting in the change of water diffusion capacity and tissue oxygen consumption.

The pathogenesis of tubulointerstitial disease lies in damage of tubules, changes of inflammation, edema or subsequent fibrosis involving corresponding interstitial regions, and following regulation of intra-renal microcirculation, while glomeruli are initially intact[16]. Besides, tubulointerstitial changes subsequent to kinds of glomerular diseases also play a key role of disease progress. This is a perfect disease model for initial study of functional MR characteristics. The aim of this study was to observe the characteristics of BOLD and DW magnetic resonance imaging both in patients with acute and chronic tubulointerstitial nephropathy (TIN).

\section{Methods}

Patients

Patients with acute tubulointerstitial nephritis (ATIN) matched 1: 2: 1 with chronic tubulointerstitial nephritis (CTIN) and healthy control were enrolled in this study from Jan 2008 to Jan 2009. ATIN patients were included if they (a) were adults diagnosed with biopsy-proven ATIN, (b) were capable to undergo fMR examination three days within percutaneous renal biopsy, (c) had no signs of other kidney diseases both clinically and pathologically. CTIN patients were selected from our specialty clinic for all-cause tubulointerstitial nephritis (TIN) diseases. Patients were included if they (a) were adults clinically diagnosed with CTIN, (b) clinically had no signs of other kidney diseases, and been followed up for more than one year, (c) with a stable serum creatinine level at CKD stage 2-5 [17]and well-controlled hemoglobin level in the recent three months. Healthy volunteers were recruited if they (a) were adults with no history of renal or cardiac diseases, and (b) had normal serum creatinine concentrations one week before MR scanning. Patients with renal malignancy, malformation, and history of partial nephrectomy were excluded from the study. For ATIN patents, serum creatinine (SCr) and hemoglobulin were routinely performed for six months (T6). BOLD and DW imaging were performed at the time of renal biopsy(TO) and the third month after supportive therapy(T3).

This prospective study was in compliance with the declaration of Helsinki, and approved by the Human Ethics Committee of Peking University First Hospital. All subjects provided written informed consent and were compatible with MR scanning.

MR imaging

All patients underwent MR imaging with a 3.0-T MR scanner (General Electric Medical Systems, Milwaukee, WI, USA). A multiple gradient-echo (mGRE) sequence was used to acquire $12 \mathrm{~T} 2{ }^{*}$-weighted images for calculation of $\mathrm{R}_{2}$ * map. The parameters of sequence were as follows:TR/TE/Flip angle/BW/matrix/ thickness/gap=100ms/6.7-32.1ms(12echoes)/450/31.3kHz/128×96/5mm/1mm.NEX=1, and five to 6 axial slices were acquired within one breath hold 24 seconds. DW imaging was acquired by combining a single-shot spin-echo (SE) echo planar imaging (EPI) pulse sequence and the additional motion probing gradient (MPG) pulses along the $x, y$, $z$-axes. The parameters were as follows: $\mathrm{TR} / \mathrm{TE} / \mathrm{BW} /$ matrix $=2300 \mathrm{~ms} / 56.1 \mathrm{~ms} / 250 \mathrm{kHz} / 128 \times 128$. NEX=2, and the slice position was identical to the BOLD imaging by the "copy" function embedded in the MR scanner, which were scanned within 18 seconds. We used two different b value group:0 and 200 s/mm² as well as 0 and $800 \mathrm{~s} / \mathrm{mm}^{2}$. Axial images were acquired for both BOLD and DW images.

Both $\mathrm{R}_{2}$ * map and apparent diffusion coefficient ADC map were generated on an AW 4.2 workstation (General Electric Medical Systems, Milwaukee, WI, USA) using "Functool" software. The reader was blinded to the subject's clinical information. At least 8 regions of interest (ROIs), each area of which was at least 10 pixels, were carefully placed on the cortex and medulla on the corresponding anatomical template separately (using image of TE=32.1ms as a template), the measured slices covered most part of the kidney. Because of the poor resolution of the images, particularly in severe renal impairment patients, it was not possible to reliably discriminate between the cortex and the medulla, which meant that the ROls could not be reliably placed. Hence it was only possible to calculate global ADCs for each kidney. The ROIs were manually delineated in the parenchyma of the kidneys. Both $R_{2}{ }^{*}$ and $A D C$ values were read out on the corresponding $R_{2}{ }^{*}$ and ADC map [Fig 2-3]. The cortical and medullary $\mathrm{R}_{2}$ * as well as global $A D C$ of kidney were calculated separately for each side.

Pathology

Renal tissues from four ATIN patients were handled routinely by Haemotoxylin-Eosin, Masson's trichrome, periodic acid-Schiff, and periodic acidsilver methenamine staining for light microscopy examination. The tissue core was often obtained at depths of about $1 \mathrm{~cm}$. The histopathological indexes included tubular injuries (tubular epithelial cells atrophy, vacuolar degeneration, brush border shedding, necrosis and tubulitis) and interstitial changes (edema, inflammation and fibrosis). Area and degree of tubular brush border shedding, atrophy and interstitial change were semiquantitatively assessed as scores 1, 2, 3 and 4 corresponding to not, mild, moderate and severe changes by two difference pathologists referring to a modification of the Banff Working Classification[20-21]. They were also blind to the clinical data. The activity index 
was the total of the scores for tubular injuries, interstitial edema, and inflammatory infiltration. The chronicity index was the total of the scores for tubular atrophy and interstitial fibrosis.

Statistical analysis

Statistical analyses were performed using the software SPSS Version 20.0 (IBM Corp., Armonk, NY). Data were presented as the median and range. Differences between groups were analyzed by the non-parametric Kruskal-Wallis test. Correlations were assessed according to the Pearson test for parametric data and the Spearman test for non-parametric data. The correlations between serum creatinine, eGFR levels and the fMR parameters (kidney volume, $A D C$ value and $R_{2}$ * value) of all kidneys were determined. The correlations between pathological indexes and fMR parameters were also analyzed. A P-value less than 0.05 was defined as statistically significant.

\section{Results}

Clinical and pathological characteristics

There were altogether 20 individuals recruited for this study, including 5 ATIN patients, 15 CTIN patients, and 5 healthy control. Four patients were excluded from the study because the quality of their fMR images was poor to be used(Fig 1. flow chart). Four patients with ATIN, thirteen patients with CTIN, and five healthy control were finally enrolled. The demographic and clinical data of the subjects are summarized in table 1. The ATIN patients were $43.8 \pm 19.4$ years old, with an averaged $52.0 \pm 13.3$ years old of CTIN patients. All the ATIN patients experienced acute kidney injury (AKI) defined using the Kidney Disease: Improving Global Outcomes (KDIGO)[20-21] criteria and consensus report of the Acute Disease Quality Initiative 16 Work group. There were two patients in AKI stage 1, two in AKI stage 2 and one in AKI stage 3. Renal pathology revealed that in ATIN kidneys, the glomeruli were relatively intact. Focal or diffuse tubular injuries, and diffuse interstitial edema and mononuclear cells infiltration were predominant pathological findings[Fig 4]. The activity index was averaged $12.8 \pm 3.3$, with the chronicity index $3.5 \pm 0.6$. The Scr level was $112 \sim 401 \mu \mathrm{mol} / \mathrm{I}\left(217.4 \pm 126.4 \mu \mathrm{mol} / \mathrm{l}\right.$, eGFR $\left.37.4 \pm 31.5 \mathrm{ml} / \mathrm{min} / 1.73 \mathrm{~m}^{2}\right)$ at the time of renal biopsy,and gradually declined to normal level after short-term steroids administration during the following three months, the eGFR was averaged $65.5 \pm 29.0 \mathrm{ml} / \mathrm{min}, 74.4 \pm 41.1 \mathrm{ml} / \mathrm{min}$ at the third (T3) and sixth month (T6). The hemoglobulin of ATIN patients was $99.0 \pm 13.4 \mathrm{~g} / \mathrm{L}$ initially, and corrected to $129.0 \pm 13.2 \mathrm{~g} / \mathrm{L}$.

Thirteen patients with CTIN were from our out-patient specialty for tubulointerstitial nephritis under integrative supportive therapy for CKD. The renal function was stable the recent three months before MR imaging. Patients were in CKD stage 2 5 non-dialysis, whose eGFRs were averaged $34.7 \pm 21.9 \mathrm{ml} / \mathrm{min}(\mathrm{Scr} 102 \sim 526 \mu \mathrm{mol} / \mathrm{I})$. Renal anemia of CTIN patients had already been corrected to $126.8 \pm 16.8 \mathrm{~g} / \mathrm{L}, \mathrm{which}$ was matchable with healthy control.

Functional MR imaging features

In control kidneys[Fig 5], the outline was smooth and there was clear differentiation between renal cortex and medulla on T1-weighted SE and IR sequence. The averaged volume of kidneys was $(137.8 \pm 26.6) \times 10^{3} \mathrm{~mm}^{3}$. Global ADC values of DW imaging was $3.54 \pm 0.25$ and $2.24 \pm 0.19$ respectively when $b$ value was 0,200 or $0,800 \mathrm{sec} / \mathrm{mm}^{2}$. Cortical $\mathrm{R}_{2}$ * value calculated from $\mathrm{BOLD} \mathrm{MR}$ imaging was $19.4 \pm 1.9 \mathrm{~Hz}$, that was obviously lower than medulla [(31.9 \pm 4.1$)$ vs. $(19.4 \pm 1.9) \mathrm{Hz}, \mathrm{p}<0.05]$.

In ATIN patients, swollen kidneys were observed [Fig 6]. The volume was $(176.8 \pm 82.8) \times 10^{3} \mathrm{~mm}^{3}$. ADC values obtained in DW imaging both when $b$ value $0,200 \mathrm{sec} / \mathrm{mm}^{2}$ and $0,800 \mathrm{sec} / \mathrm{mm}^{2}$ were used, were all found to have significant decreases as $23.5 \%, 19.2 \%$ respectively than control group when $b$ value was 0,200 , and $0,800 \mathrm{sec} / \mathrm{mm}^{2}$ (Table 1). And obvious rising of decreased ADC values were observed following improvement of renal function to achieve a $48.1 \%, 80.9 \%$ recovery at the third month but still lower than control (see Table 1 ). Both cortical and medullary $R_{2}$ * values of ATIN kidneys were also lower than controls at the time of renal biopsy, the differences was significant in $M R_{2}{ }^{*}$ values as 23.8\% (difference in $\mathrm{CR}_{2}$ * values was $9.3 \%$ ). The medullary $\mathrm{R}_{2}$ * values firstly went back to a level similar as control, while the cortical $\mathrm{R}_{2}$ * values remained low. For CTIN patients [Fig 7], extremely shrink kidneys with irregular outlines were found (fig 2). The volume was $89.0 \pm 23.0 \times 10^{3} \mathrm{~mm}^{3}$. Both ADC values when $b$ value $0,200 \mathrm{sec} / \mathrm{mm}^{2}$ and $b$ value $0,800 \mathrm{sec} / \mathrm{mm}^{2}$ were similar as healthy control. In $\mathrm{R}_{2}{ }^{*}$ map, medullary $\mathrm{R}_{2}{ }^{*}$ value of CTIN kidneys was averaged $28.0 \pm 5.0 \mathrm{~Hz}$, which was lower than control but the difference was not statistically significant.

Further analysis disclosed that neither ADC values nor $\mathrm{R}_{2}{ }^{*}$ values, was correlated to histopathological indexes including tubular injuries (tubular epithelial cells atrophy, vacuolar degeneration, brush border shedding, necrosis and tubulitis) and interstitial changes (edema, inflammation and fibrosis) when compared separately. We also found no relationship within $A D C$ values, $R_{2}$ * values, $A l$ and $C l$. It seems that $A D C$ and $R_{2}{ }^{*}$ values changed along with that of renal function (Table 1) in ATIN kidneys, while close relationship was only identified in MR ${ }^{*}$ values with eGFR $(R=0.682, P=0.001)$, the situation was similar whether for CTIN patients $(R=0.615, P=0.025)$ or all TIN patients $(R=0.956, P=0.044)$. Both $A D C$ values were inversely correlated to the change of $A D C$ values $(\triangle A D C$, the change of $A D C$ value over the following three months) when $b$ value was $0,200 \mathrm{sec} / \mathrm{mm}^{2}(\mathrm{R}=-0.956, \mathrm{P}=0.044)$ and $0,800 \mathrm{sec} / \mathrm{mm}^{2}(\mathrm{R}=0.968, \mathrm{P}=0.032)$. The change of medullary $R_{2}{ }^{*}$ values $\left(\Delta M R_{2}{ }^{*}\right.$, the change of

Page $4 / 11$ 
$\mathrm{MR}_{2}$ * value over the following three months) was found to have close relationship with $\triangle \mathrm{ADC}$ when $b$ was $0,200 \mathrm{sec} / \mathrm{mm} \mathrm{m}^{2}(\mathrm{R}=0.979, \mathrm{P}=0.021)$ which was regarded as a marker mainly affected by blood perfusion, inversely with pathological $\mathrm{Cl}(\mathrm{R}=-0.977, \mathrm{P}=0.023)$. Renal long-term prognosis analysis among candidate predictive markers showed that no relationship was found with time-point $A D C$ or $R_{2}{ }^{*}$ values, but $\triangle M R_{2}{ }^{*}$ had a significant correlation to Scr levels at the third $(R=-0.959, P=0.041)$ and sixth month $(R=0.98, P=0.02)$. In other words, the lower the $A D C$ value ( $b$ was $0,200 \mathrm{sec} / \mathrm{mm}^{2}$ ), the greater the increase of $A D C$ in the next three months, the greater the increase of $M R_{2}{ }^{*}$, then the subsequent Scr level would be lower.

in ATIN kidneys, a significant reduction of medullary $R_{2}{ }^{*}$ value and ratio of $M R_{2}{ }^{*}$ to $\mathrm{CR}_{2}{ }^{\star}$ were detected. The rapid and reversible change of the medullary $\mathrm{R}_{2}$ * values uggested that the tubular injury is mainly caused by ischemic factors.

Although both $\mathrm{CR}_{2}{ }^{*}$ and $\mathrm{MR}_{2}{ }^{*}$ were decreased than those of healthy control at ATIN, their changes after treatment were varied, with further decline of $\mathrm{CR}_{2}$ * in two patients; in the CTIN group, only a low level of $\mathrm{MR}_{2}$ * was observed while $\mathrm{CR}_{2}$ * could remain at the normal level, suggesting that there might be a delayed recovery of AKD injury and the "pseudo normalization" of $\mathrm{CR}_{2}{ }^{*}$ caused by oxygen adaptation changes during CKD.

\section{Discussion}

In this study, we assessed kidneys of ATIN presenting acute kidney injury and CTIN kidneys of stable renal function with DW and BOLD MR imaging. The correlation of functional MR parameters with critical pathological and clinical factors indicate the potential significance of these novel techniques as noninvasive methods, contributing to diagnosis, long-term prognosis assessment and further understanding of kidney diseases.

Diffusion-weighted MR imaging yields the ADC value as an index reflecting microenvironment of diffusing water molecules. It is considered as a simple marker reflecting tissue microstructure. In case of renal dysfunction, tubular injuries lead to reduced water reabsorption process resulting in decreased diffusion[22]. Factors involving microcapillary perfusion, status of tissue edema and fibrosis also theorically dedicate to ADC. Boor $P$ et al [10] observed ADC values in unilateral ureteral obstruction rat model, and found that renal ADCs postmortem dropped almost $75 \%$ off than baseline in vivo, suggested the leading role of perfusion contributing to ADC value especially when $b$ value $<200$ sec/mm ${ }^{3}$. Oppositely when $b$ level was higher than $400 \mathrm{sec} / \mathrm{mm}^{3}, A D C$ value declined only $25 \%$, because the value was mostly generated from the effect of diffusion. Xu $Y$ et al [23] reported that ADC values of impaired kidneys (when $b$ was 500) were significantly lower in a linear but positive correlation with eGFR in patients mainly with renal arterial stenosis. In the current study, we revealed similar level of the global ADC values in CTIN patients as control. While there were obviously decreased but reversible changes of ADC values when $b$ is 0,200 and 0,800 sec/mm in patients with ATIN, when compared with control. Presumably this indicated that there was redistribution of intra-renal micro-circulation in the background of interstitial inflammation leading to reduced blood perfusion, and decreased water diffusion because of tubular injuries, thus ADC in both low and higher $b$ values declined. The delayed recovery of ADC (especially when $b$ was $0,200 \mathrm{sec} / \mathrm{mm}^{2}$ ) also meant that kidney injury was still in the process of repair or left behind chronicity. After that, the kidney was likely to undergo adaptive changes continually, and when it finally reached a stable CKD stage, as data shown in CTIN patients, ADC could approach normal level. It is concluded from the current study that ADC may serve as a promising marker reflecting reversible acute injury, but not sensitive for interstitial fibrosis.

As we have known, the $\mathrm{R}_{2}$ * value of BOLD MR imaging has been regarded as a factor reflecting tissue oxygenation[24]. The renal medulla is vulnerable to hypoxia because of low oxygen delivery due to low vascular density in medulla, arterial-venous shunting, and high oxygen consumption for active transcellular transport of sodium and chloride in loop of Henle. Therefore, it is not difficult to understand that oxygen consumption and $\mathrm{R}_{2}{ }^{*}$ values decrease due to renal tubulointerstitial injury. If the injury is mainly related to direct toxicity, it only shows the decrease of cortical $R_{2} \star[25]$. However, if the mechanism of renal tubular injury is also involved in ischemic factors, such as renal ischemia with redistribution of blood flow during acute inflammation, renal artery stenosis, diabetic microangiopathy, acute rejection of graft-kidney[26] and aristolochic acid nephropathy, then the $\mathrm{R}_{2}$ * of both cortex and medulla will change. The change of medullary $\mathrm{R}_{2}{ }^{*}$ would happen earlier and more significant. In our study, we verified that medullary $\mathrm{R}_{2}$ * was closely correlated to eGFR for all the patients, that it can be regarded as an important marker to reflect renal dysfunction. The change of $\mathrm{CR}_{2}{ }^{*}$ was mild, it is because acute ischemia compromises oxygen delivery while oxygen consumption by the reabsorption is maintained. The reduction of $\mathrm{MR}_{2}$ * to $\mathrm{CR}_{2}$ * ratio was detected in ATIN, suggested that the tubular injury was mainly caused by ischemic factors. The delayed recovery of $\mathrm{CR}_{2}{ }^{*}$ value is due to the increase of tissue oxygenation due to the improvement of blood perfusion, so the change of $\mathrm{CR}_{2}{ }^{*}$ was not significant. It is interesting that cortical $\mathrm{R}_{2}{ }^{*}$ was normalized in CTIN kidneys opposite to the persistent low medullary $\mathrm{R}_{2}{ }^{*}$. And this "pseudo normalization" of $\mathrm{CR}_{2}{ }^{*}$ by supportive change in CTIN may be the result of the aggravation of renal ischemic changes, and contributes to the progression of CKD.

DW MR imaging and BOLD imaging are the basic classical sequences of functional nuclear magnetic resonance in clinical application, but the exploration in the field of non-transplanted kidney disease is still limited. From the results of this study, we consider that DW combined with BOLD imaging may be helpful to judge AKD, especially the emergence of AKD on the basis of the original CKD in case of not applicable to kidney

Page 5/11 
biopsy, as a non-invasive diagnostic method that can be tried. However, the response of DW MR to renal interstitial fibrosis is insensitive, because the result of patients with stage CKD3-5 showed no difference from those of normal. But the joint examination using the basic scanning sequence is one of the feasible ways that can be popularized and applied to clinic in the future.

The limitation of our study was the disability to get direct information of intra-renal perfusion, despite that DW and BOLD imaging were nowadays the most frequently used functional MR imaging techniques in human diseases. Combination of these data could indirectly to some extent reflect tissue edema, inflammation, fibrosis and even be relevant to organ dysfunction. Microcirculation might also be roughly assessed, but could not be determined histologically by routine methods. More and special multi-sequence should be researched and developed in the future in order to further understand the mechanism of diseases[27-28]. The limitation of functional MR sequence scanning also lies in the influence of respiration on the scanning image quality. For critically ill patients, the poor respiratory cooperation in the intensive care unit is a problem that restricts the clinical application of fMR.

This is an initial observational study and we produced direct evidence of intra-renal oxygenation and got global values of ADC in acute and chronic TIN patients for the first time. Short-time reduced $\mathrm{R}_{2}$ * values of both cortical and medullary accompanying with reduced $A D C$ values were observed in ATIN kidneys. On the contrary, there was obvious but persistent change of tissue oxygenation in CTIN kidneys. Long-term observation deserves close attention.

\section{Abbreviations}

MR: magnetic resonance; fMR: functional MR; DW:diffuse weighted; BOLD: Blood oxygen level-dependent; ADC: global apparent diffusion coefficient; ATIN: acute tubulointerstitial nephritis; CTIN:chronic tubulointerstitial nephritis; $\mathrm{CR}_{2}{ }^{*}$ : cortical $R_{2}{ }^{*}$ values; $M R_{2}{ }^{*}: m_{\text {medullary }} R_{2}{ }^{*}$ values

\section{Declarations}

\section{Acknowledgements}

We gratefully thank Dr. Ju Cao and Dr. Gang Liu for her help of data collection, and Dr. Xiaomei Li for the valuable suggestion of project design. We also thank the patients for agreeing to participate in this study.

\section{Authors' Contributions}

TS and LY contributed to patient diagnosis, management and clinical data analysis. XY, RW and XW contributed to analysis of functional MR imaging data and provided relating images. TS and XY wrote manuscript drafting, contributed to data analysis, interpretation and intellectual content of critical importance to the work described. LY and XW interpretation and intellectual content of critical importance to the work and revised the manuscript. All authors had the opportunity to revise the manuscript.

\section{Funding}

This research was supported by grants from National Science and Technology Major Projects for major new drugs innovation and development (2017ZX09304028), the Beijing Young Scientist Program (BJJWZYJH01201910001006) and Peking University Clinical Scientist Program. The funders played roles in the collection of data, follow-up of the patients and interpretation of data.

\section{Availability of data and materials}

The patient was regularly followed up and the clinical data is traceable. The datasets used and analysed during the current study are available from the corresponding author on reasonable request.

\section{Ethics approval and consent to participate}

Not Applicable.

\section{Consent for Publication}

Written informed consent was obtained from the patients for publication and any accompanying images.

\section{Competing interests}

No one of the Authors has a financial and non-financial competing interest.

\section{References}


1.Zhang JL, Rusinek H, Chandarana H, Lee VS. Functional MRI of the kidneys. J Magn Reson Imaging. 2013,37: 282-293.

2.Prasad PV. Functional MRI of the kidney: tools for translational studies of pathophysiology of renal disease.Am J Physiol Renal Physiol. 2006,290: F958-F974

3. Caroli A, Schneider M, Friedli I, Ljimani A, De Seigneux S, Boor P, et al. Diffusion-weighted magnetic resonance imaging to assess diffuse renal pathology: a systematic review and statement paper. Nephrol Dial Transplant. 2018,33: ii29-ii40

4.Dong J, Yang L, Su T, Yang X, Chen B, Zhang J, Wang X, et al. Quantitative assessment of acute kidney injury by noninvasive arterial spin labeling perfusion MRI: a pilot study. Sci China Life Sci, 2013, 56: 745-750

5.Zhou HY, Chen TW, Zhang XM. Functional Magnetic Resonance Imaging in Acute Kidney Injury: Present Status. Biomed Res Int. 2016;2016:2027370.

6.Mao W, Zhou J, Zeng M, Ding Y, Qu L, Chen C, et al. Chronic kidney disease: Pathological and functional evaluation with intravoxel incoherent motion diffusion-weighted imaging. J Magn Reson Imaging. 2018, 47: 118-124

7. Wang ZJ, Kumar R, Banerjee S, Hsu CY. Blood oxygen level-dependent (BOLD) MRI of diabetic nephropathy: preliminary experience. J Magn Reson Imaging. 2011,33: 655-660

8.Palmucci S, Mauro LA, Veroux P, Failla G, Milone P, Ettorre GC, et al. Magnetic resonance with diffusion-weighted imaging in the evaluation of transplanted kidneys: preliminary findings.Transplant. Proc. 2011,43:960-966

9.Tewes S, Gueler F, Chen R, Gutberlet M, Jang MS, Meier M, et al. Functional MRI for characterization of renal perfusion impairment and edema formation due to acute kidney injury in different mouse strains.PLoS ONE. 2017,12:1-18

10.Boor P, Perkuhn M, Weibrecht M, Zok S, Martin IV, Gieseke J, et al. Diffusion-weighted MRI does not reflect kidney fibrosis in a rat model of fibrosis. J Magn Reson Imaging. 2015, 42:990-998

11. Hofmann L, Simon-Zoula S, Nowak A, Giger A, Vock P, Boesch C, et al. BOLD-MRI for the assessment of renal oxygenation in humans: Acute effect of nephrotoxic xenobiotics. Kidney Int. 2006, 70: 144-150

12. Hueper K, Rong S, Gutberlet M, Hartung D, Mengel M, Lu X, et al. T2 relaxation time and apparent diffusion coefficient for noninvasive assessment of renal pathology after acute kidney injury in mice: comparison with histopathology. Investigative Radiology. 2013,48:834-842.

13. Li LP, Lu J, Zhou Y, Papadopoulou MV, Franklin T, Bokhary U, et al. Evaluation of intrarenal oxygenation in iodinated contrast-induced acute kidney injury-susceptible rats by blood oxygen level-dependent magnetic resonance imaging. Investigative Radiology. 2014,49:403-410.

14.Michaely HJ, Metzger L, Haneder S, Hansmann J, Schoenberg SO, Attenberger UI. Renal BOLD-MRI does not reflect renal function in chronic kidney disease. Kidney Int. 2012,81:684-689 .

15. Nangaku M. Chronic hypoxia and tubulointerstitial injury: a final common pathway to end-stage renal failure.J Am Soc Nephrol. 2006,17:1725.

16. Su T, Gu Y, Sun P, Tang J, Wang S, Liu G, et al. Etiology and renal outcomes of acute tubulointerstitial nephritis: a single-center prospective cohort study in China. Nephrol. Dial. Transplant. 2018,33:1180-1188

17. Levey AS, Stevens LA, Schmid CH, Zhang YL, Castro AF, Feldman HI. A new equation to estimate glomerular filtration rate. Ann Intern Med. 2009;150:604-612.

18. Solez K, Colvin RB, Racusen LC, Haas M, Sis B, Mengel M, et al. Banff 07 classification of renal allograft pathology: updates and future directions. Am J Transplant. 2008,8:753-760.

19.Racusen LC, Solez K, Colvin RB, Bonsib SM, Castro MC, Cavallo T, et al. The Banff 97 working classification of renal allograft pathology. Kidney Int. 1999;55:713-723.

20.Kidney Disease: Improving Global Outcomes (KDIGO) Acute Kidney Injury Work Group. KDIGO practice guideline for acute kidney injury. Kidney Int suppl. 2012;2:1-138.

21.Chawla LS, Bellomo R, Bihorac A, Goldstein SL, Siew ED, Bagshaw SM, et al. Acute kidney disease and renal recovery: consensus report of the Acute Disease Quality Initiative (ADQI) 16 Workgroup. Nat Rev Nephrol. 2017;13:241-257. 
23.Xu Y, Wang X, Jiang X. Relationship Between the Renal Apparent Diffusion Coefficient and Glomerular Filtration Rate: Preliminary Experience. J Magn Reson Imaging. 2007,26:678-681.

24.Inoue T, Kozawa E, Okada H, Inukai K, Watanabe S, Kikuta T, et al. Noninvasive evaluation of kidney hypoxia and fibrosis using magnetic resonance imaging. J Am Soc Nephrol. 2011 Aug;22(8)

25.Cao J, Yang XD, Wang XY, Qu L, Liu G, Li XM. Differential changes of intrarenal oxygenation in rat models of acute tubular necrosis caused by aristolochic acid and gentamicin. Zhonghua Yi Xue Za Zhi 2010, 90:1208-1212

26.Sadowski EA, Fain SB, Alford SK, Korosec FR, Fine J, Muehrer R, et al. Assessment of acute renal transplant rejection with blood oxygen leveldependent MR imaging: initial experience. Radiology 2005 Sep;236(3)

27.Wang W, Yu Y, Wen J, Zhang M, Chen J, Cheng D,et al. Combination of Functional Magnetic Resonance Imaging and Histopathologic Analysis to Evaluate Interstitial Fibrosis in Kidney Allografts. Clin J Am Soc Nephrol, 2019,14:1372-1380

28.Liang L, Chen WB, Chan KW, Li YG, Zhang B, Liang CH, et al. Using intravoxel incoherent motion MR imaging to study the renal pathophysiological process of contrast-induced acute kidney injury in rats: comparison with conventional DWI and arterial spin labelling. Eur Radiol 2016;26:1597-1605

\section{Tables}

Table 1. Laboratory and fMR data of ATIN and CTIN kidneys

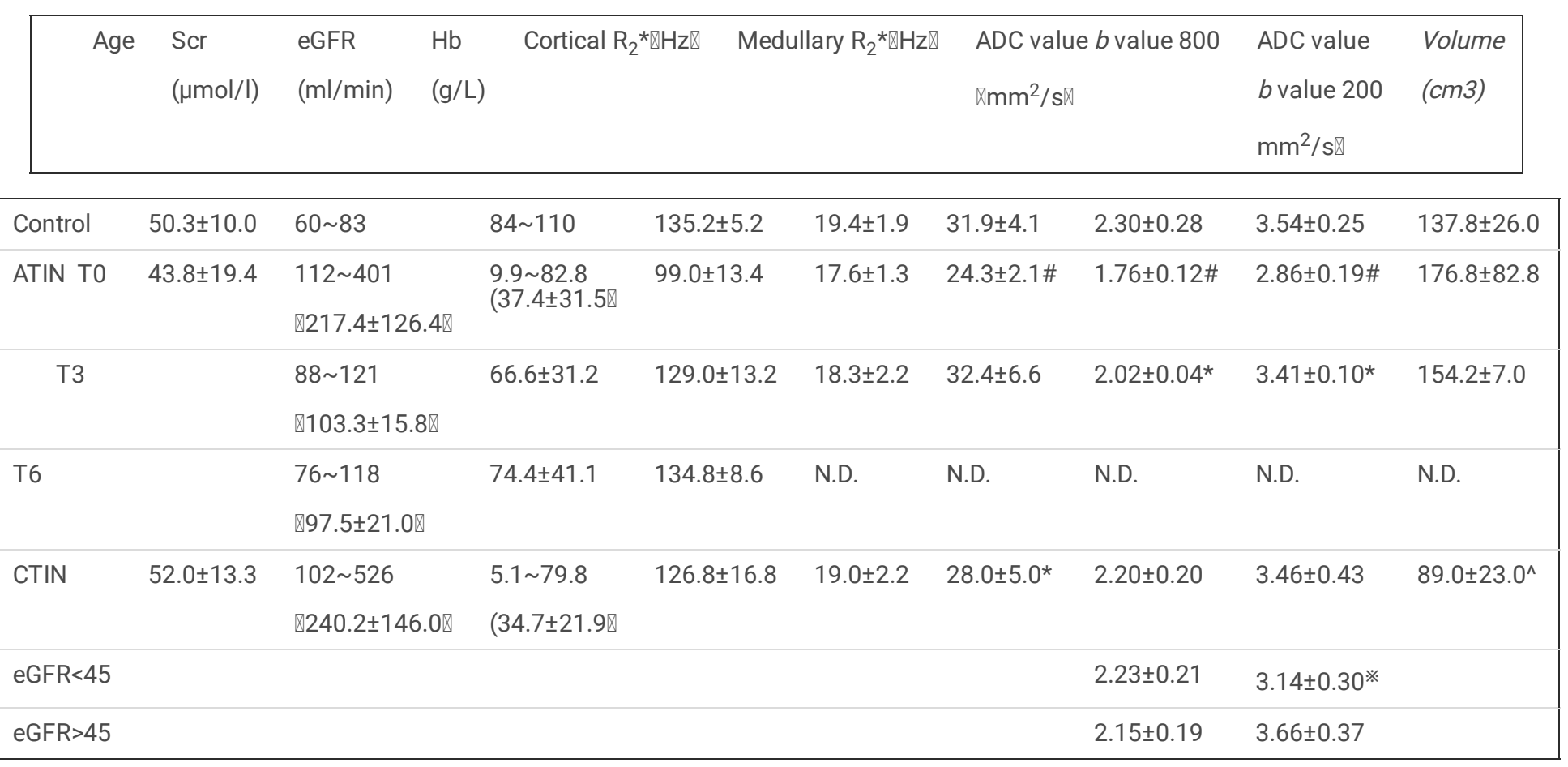

Note: \# when compared between T0 and control, the difference is significant $P<0.05$.

* when compared between T0 and T3, the difference is significant $P<0.05$.

no significant difference was found between T3 and control

${ }^{\wedge}$ when compared with control, the difference is significant $\mathrm{P}<0.05$.

※when compared with group eGFR $>45 \mathrm{ml} / \mathrm{min}$, the difference is significant $\mathrm{P}<0.05$.

\section{Figures}




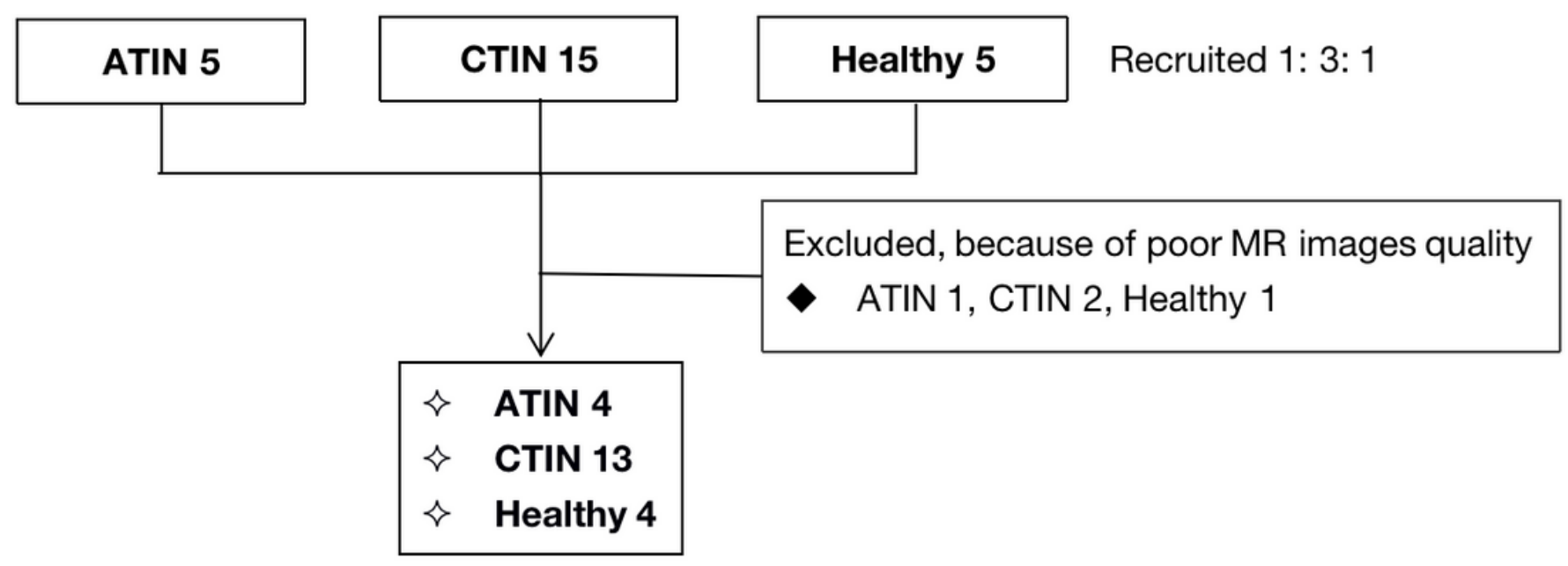

Figure 1

the flow chart of the study.
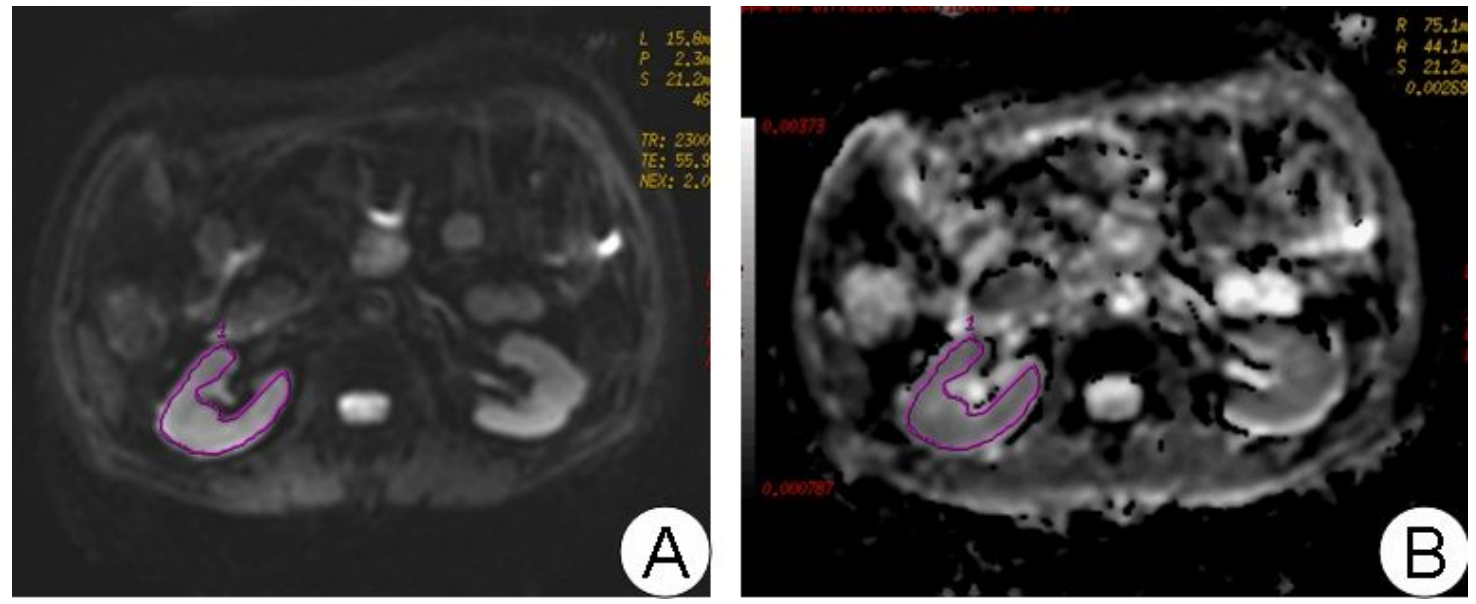

Figure 2

A:DWI image. B: the corresponding ADC diagram. The method of manual placement of ROI is used to draw the outline of the kidney on the anatomical map with (DWI).
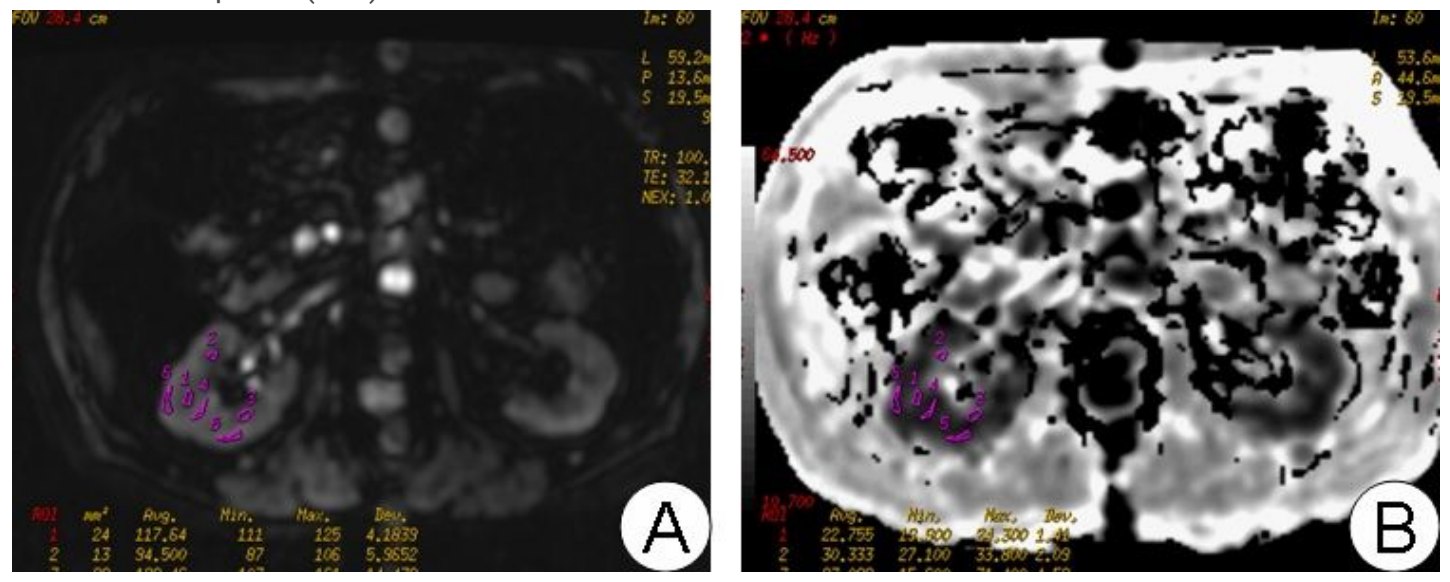

Figure 3 
A:BOLD image anatomical template. BRV R2* figure. The method of manually placing ROI on the anatomical template is to place at least three $\mathrm{ROI}$, in the cortical medulla and read the corresponding R2* value on the corresponding R2* diagram.

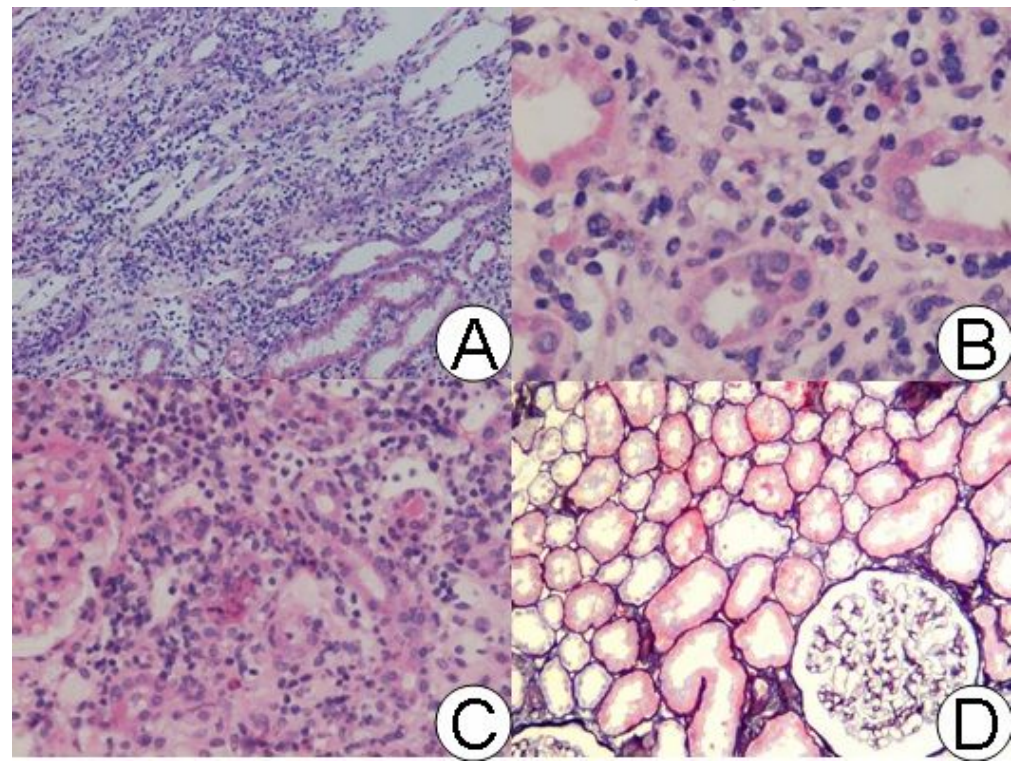

\section{Figure 4}

pathological pictures of ATIN and normal kidney (all HE staining). A: example 1, magnification 100xdexB: case 2, magnification x400 × C: case 3, magnification $x 200$. From A to C, ATIN, showed exfoliated brush margin of renal tubules, dilated lumen, diffuse edema of renal interstitium, multifocal or diffuse (C) lymphoid and monocytes infiltration and eosinophils infiltration. There were no obvious pathological changes in glomeruli and arterioles. Figure D shows normal kidney, magnification x100: glomeruli and tubules are normal.

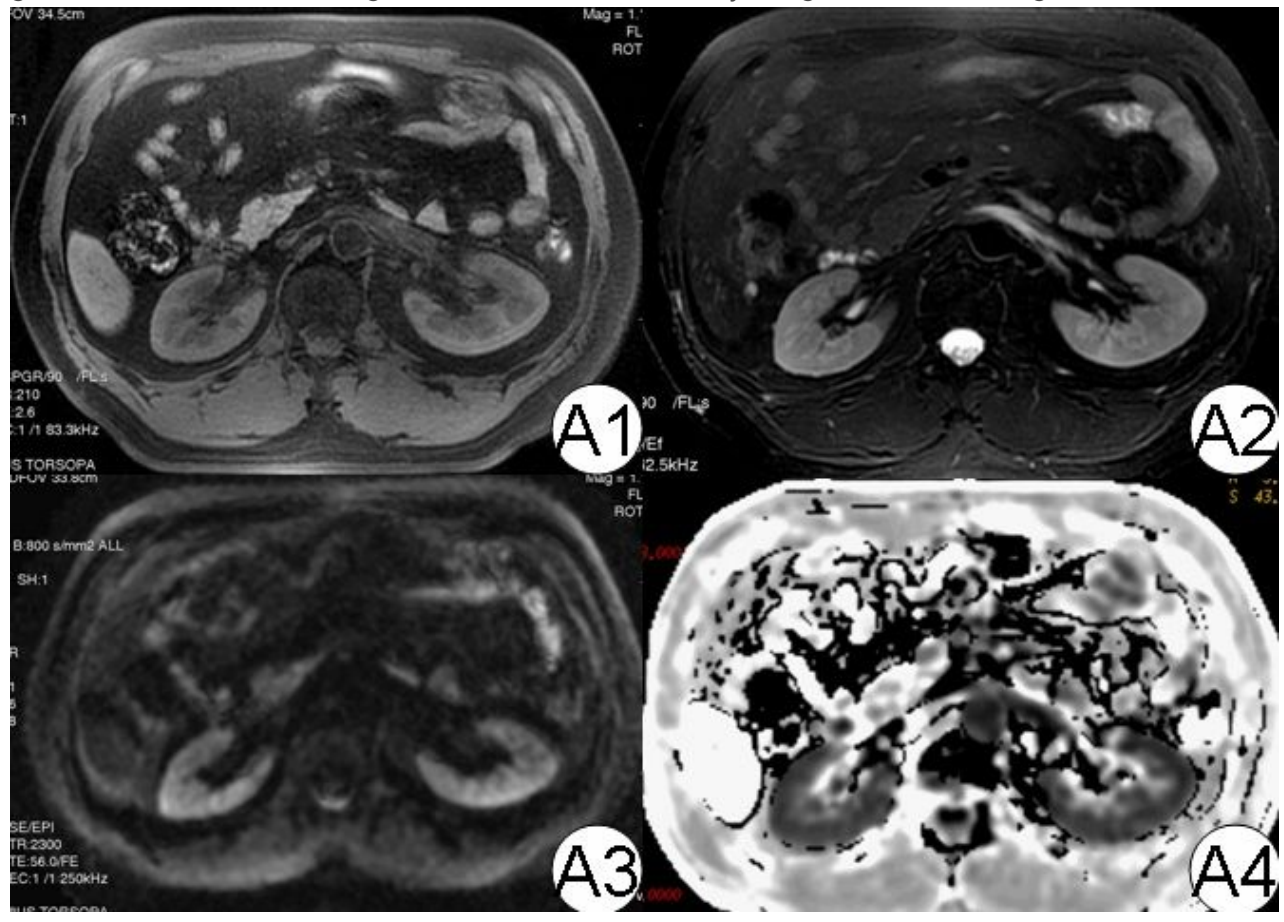

Figure 5

MRI diagram of normal kidney. In the picture, A1 to A4 are T1WI, T2WI, DWI and R2*, respectively. It can be seen that the demarcation of the epithelium and medulla on T1WI, T2WI and R2 * maps is clear (3 points). 


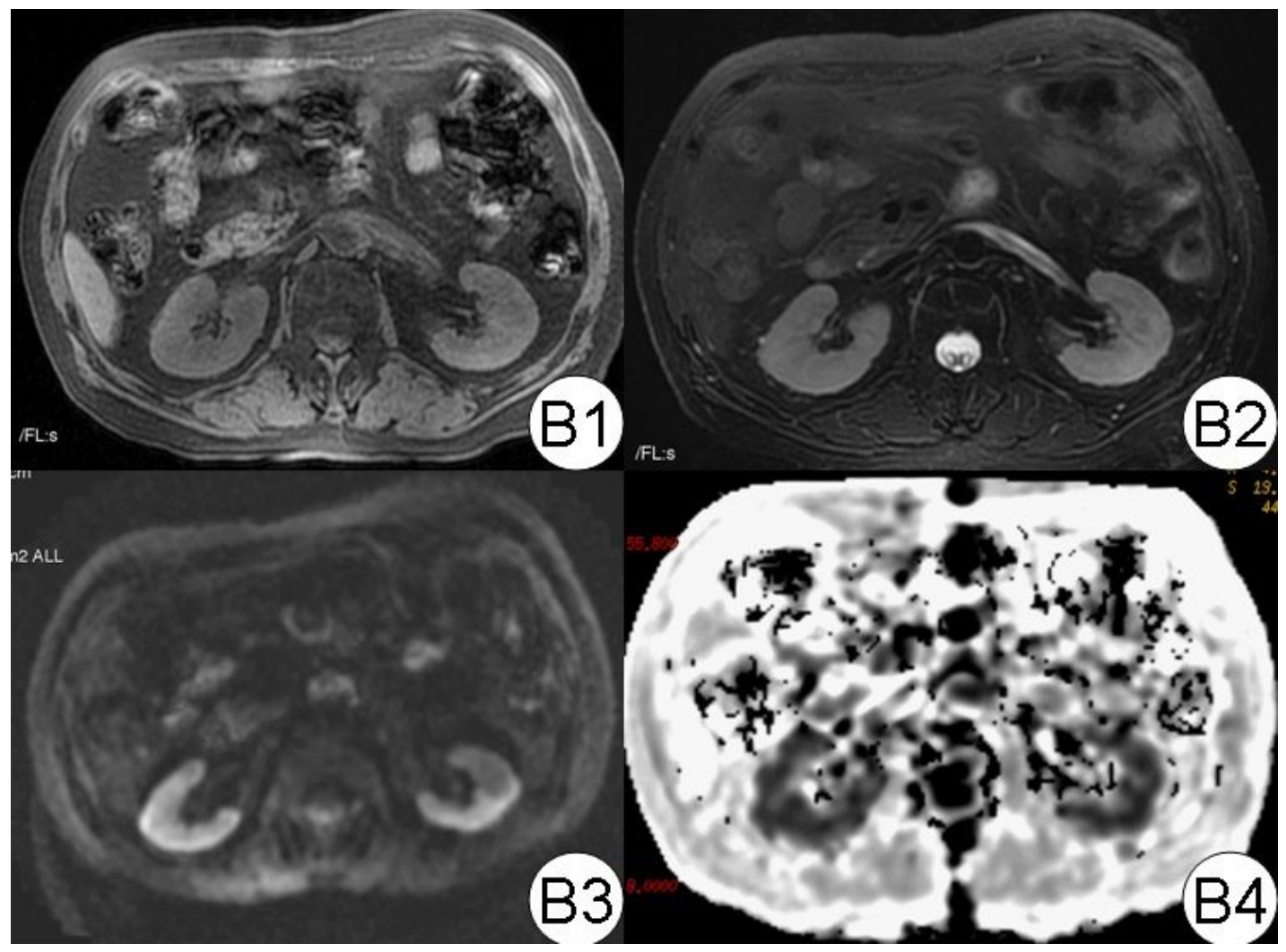

Figure 6

ATIN kidney MRI diagram. In the picture, B1 to B4 are T1WI and T2WI DWI and R2* pictures, respectively. It can be seen that the corticomedulla boundary between T1WI and T2WI is OK (2 points). Compared with normal, the cortical area of R2 * map is slightly irregular.

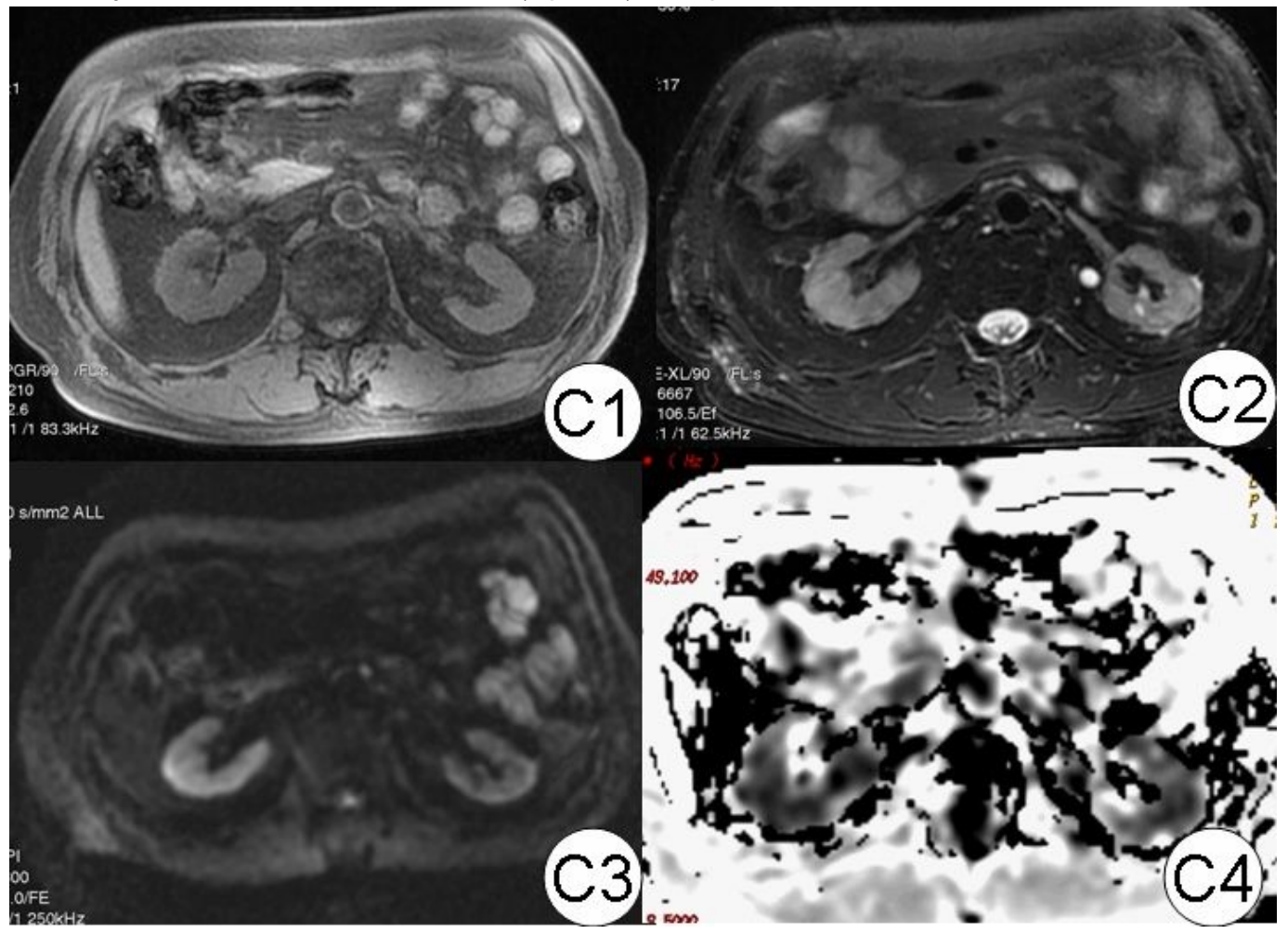

Figure 7

CTIN kidney MRI diagram. In the figure, C1 to C4 are T1WI and T2WIMagol DWI and R2* pictures, respectively. The epithelial medulla boundary of T1WI and T2WI could only be seen faintly (1 point). Compared with normal and ATIN, the epithelial medulla of R2 * was obviously irregular. 